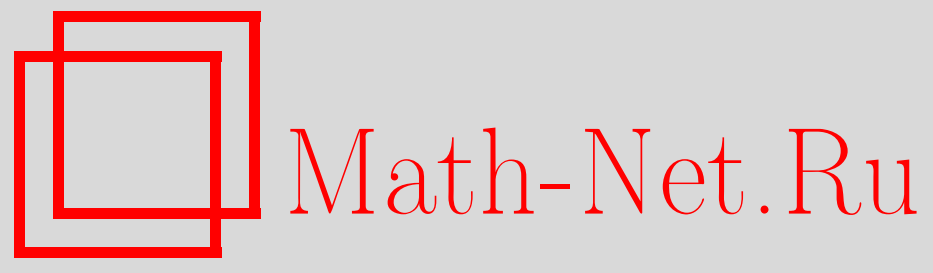

Т. К. Юлдашев, А. И. Середкина, Обратная задача для квазилинейных интегро-дифференциальных уравнений в частных производных высокого порядка, Вестн. Сам. гос. техн. ун-та. Сер. Физ.мат. науки, 2013, выпуск 3(), 46-55

DOI: https://doi.org/10.14498/vsgtu1133

Использование Общероссийского математического портала MathNet.Ru подразумевает, что вы прочитали и согласны с пользовательским соглашением

http://www.mathnet.ru/rus/agreement

Параметры загрузки:

IP: 35.174 .16 .151

26 апреля 2023 г., 13:04:16




УДК 517.956.47

\section{ОБРАТНАЯ ЗАДАЧА ДЛЯ КВАЗИЛИНЕЙНЫХ ИНТЕГРО-ДИФФЕРЕНЦИАЛЬНЫХ УРАВНЕНИЙ В ЧАСТНЫХ ПРОИЗВОДНЫХ ВЫСОКОГО ПОРЯДКА}

\section{Т. К. Юлдашев, А. И. Середкина}

Сибирский государственный аэрокосмический университет им. ак. М. Ф. Решетнева, Россия, 660014, Красноярск, пр. газеты имени «Красноярский рабочий», 31.

E-mails: tursunbay@rambler.ru, anytik888@yandex.ru

Предлагается методика изучения обратной задачи для некоторых классов квазилинейных уравнений в частных производных высокого порядка. Доказывается теорема о существовании и единственности решения данной обратной задачи.

Ключевые слова: обратная задача, квазилинейное уравнение в частных производных, суперпозиция дифференциальных операторов, метод характеристик, существование и единственность решения.

1. Постановка задачи. С точки зрения физических приложений представляют большой интерес дифференциальные уравнения в частных производных высоких порядков. Изучение многих задач газовой динамики, теории упругости, теории пластин и оболочек приводит к рассмотрению дифференциальных уравнений в частных производных высоких порядков.

Локальная теория дифференциальных уравнений в частных производных первого порядка, основанная на понятиях производной по направлению и характеристик, начала формироваться еще в XVIII веке. Характеристики замечательны тем, что выражения в левой части уравнений в частных производных первого порядка представляют собой производную неизвестной функции по направлению вдоль характеристики. Это позволяет, перейдя к новой переменной, представить уравнение в частных производных первого порядка как обыкновенное дифференциальное уравнение, описывающее изменение неизвестной функции вдоль линии характеристики.

Основная идея, на которой основан развиваемый в статье подход, состоит в том, что выражение уравнений в частных производных высокого порядка через суперпозицию дифференциальных операторов в частных производных первого порядка позволяет применять методы решения дифференциальных уравнений в частных производных первого порядка.

В области $D \equiv D_{T} \times \mathbb{R}$ рассматривается нелинейное уравнение вида

$$
\left(\frac{\partial^{2}}{\partial t^{2}}-\frac{\partial^{2}}{\partial x^{2}}\right)^{n} L_{0}[u(t, x)]=f(t, x, \vartheta(t))
$$

с начальными

$$
\left.u(t, x)\right|_{t=0}=\varphi_{1}(x),\left.\quad \frac{\partial^{i} u(t, x)}{\partial t^{i}}\right|_{t=0}=\varphi_{i+1}(x), \quad x \in \mathbb{R}, \quad i=\overline{1,2 n}
$$

Турсун Камалдинович Юлдашев (к.ф.-м.н., доц.), докторант, доцент, каф. высшей математики. Анна Игоревна Середкина, магистрант, инженер, каф. высшей математики. 
и дополнительными

$$
\begin{gathered}
\left.\int_{0}^{t} P(t, s) u(s, x) d s\right|_{x=x_{0}}=\psi(t), \quad t \in D_{T} \\
\left.\vartheta(t)\right|_{t=0}=\varphi_{0}=\mathrm{const}
\end{gathered}
$$

условиями, где

$$
L_{0}[u(t, x)] \equiv u_{t}+a u_{x}, \quad a=a\left(t, x, \int_{0}^{T} \int_{-\infty}^{\infty} K(s, y) u(s, y) d y d s\right)
$$

$\psi(0)=0 ; u(t, x)$ и $\vartheta(t)$ - неизвестные функции; $f(t, x, \vartheta) \in C\left(D \times D_{T}\right) ; P(t, s) \in$ $C\left(D_{T}^{2}\right) ; a(t, x, u) \in C^{2 n, 2 n}(D \times \mathbb{R}) ; K(t, x) \in C(D) ; \varphi_{i}(x) \in C^{2 n+2}(\mathbb{R}), i=$ $=\overline{1,2 n+1} ; \psi(t) \in C\left(D_{T}\right) ; D_{T} \equiv[0, T], 0<T<\infty ; n$ - произвольное натуральное число.

Изучению разрешимости обратных задач для линейных дифференциальных уравнений в частных производных посвящено большое количество работ. Библиографию многих публикаций, посвященных теории линейных обратных задач, можно найти в [1-3]. В настоящей работе изучается обратная задача, где восстанавливаемая функция $\vartheta(t)$ нелинейно входит в уравнение. При решении обратной задачи (1)-(4) относительно восстанавливаемой функции получается нелинейное интегральное уравнение Вольтерра первого рода, которое с помощью неклассического интегрального преобразования сводится к нелинейному интегральному уравнению второго рода. Задание условия (4) при преобразовании обеспечивает единственность решения нелинейного интегрального уравнения первого рода и определяет значение неизвестной функции в начальной точке $t=0$, т. е. $\vartheta(0)=\varphi_{0}$. Обратные задачи для квазилинейных дифференциальных уравнений в частных производных первого порядка ранее рассматривались в работах $[4,5]$.

Определение. Решением обратной задачи (1)-(4) называется пара непрерывных функций $\left\{u(t, x) \in C^{2 n+1,2 n+1}(D), \vartheta(t) \in C\left(D_{T}\right)\right\}$, удовлетворяющая уравнению (1) и условиям (2)-(4).

2. Задача Коши (1), (2). Левую часть уравнения (1) запишем в виде

$$
\left(\frac{\partial^{2}}{\partial t^{2}}-\frac{\partial^{2}}{\partial x^{2}}\right)^{n} L_{0}[u]=\left(\frac{\partial}{\partial t}-\frac{\partial}{\partial x}\right)^{n}\left(\frac{\partial}{\partial t}+\frac{\partial}{\partial x}\right)^{n} L_{0}[u]=L_{2}^{n}\left[L_{1}^{n}\left[L_{0}[u]\right]\right]
$$

где

$L_{2}\left[L_{1}^{n}\left[L_{0}[u]\right]\right] \equiv\left(L_{1}^{n}\left[L_{0}[u]\right]\right)_{t}-\left(L_{1}^{n}\left[L_{0}[u]\right]\right)_{x}, \quad L_{1}\left[L_{0}[u]\right] \equiv\left(L_{0}[u]\right)_{t}+\left(L_{0}[u]\right)_{x}$.

Тогда уравнение (1) приобретает вид

$$
L_{2}^{n}\left[L_{1}^{n}\left[L_{0}[u]\right]\right]=f(t, x, \vartheta(t)) .
$$

Из (5) видно, что уравнение (1) имеет одну однократную характеристику:

$$
x-\int_{0}^{t} a\left(s, x, \int_{0}^{T} \int_{-\infty}^{\infty} K(\theta, y) u(\theta, y) d y d \theta\right) d s=C_{1}
$$


две $n$-кратные характеристики:

$$
x-t=C_{2}, \quad x+t=C_{3},
$$

где $C_{i}$ - произвольные постоянные, $i=1,2,3$.

Тогда, интегрируя уравнения (5) $n$ раз вдоль линии третьей характеристики, получаем

$$
\begin{gathered}
L_{2}^{n-1}\left[L_{1}^{n}\left[L_{0}[u]\right]=\Phi_{1}(x+t)+\int_{0}^{t} f(s, x, \vartheta(s)) d s\right. \\
L_{2}^{n-2}\left[L_{1}^{n}\left[L_{0}[u]\right]\right]=\Phi_{2}(x+t)+\Phi_{1}(x+t) t+\int_{0}^{t}(t-s) f(s, x, \vartheta(s)) d s \\
\vdots \\
L_{1}^{n}\left[L_{0}[u]\right]=\sum_{i=1}^{n} \Phi_{i}(x+t) \frac{t^{n-i}}{(n-i) !}+\int_{0}^{t} \frac{(t-s)^{n-1}}{(n-1) !} f(s, x, \vartheta(s)) d s
\end{gathered}
$$

где $\Phi_{i}(x), i=\overline{1, n}$ - произвольные непрерывные функции, которые подлежат дальнейшему определению.

Из (6) в силу начального условия $(2)$ имеем $\Phi_{1}(x)=\varphi_{2 n+1}$. Так как вдоль третьей характеристики $\left(x+t=C_{3}\right)$

$$
\begin{gathered}
\frac{d L_{1}^{n}\left[L_{0}[u]\right]}{d t}=\frac{\partial L_{1}^{n}\left[L_{0}[u]\right]}{\partial t}+\frac{\partial L_{1}^{n}\left[L_{0}[u]\right]}{\partial x} \frac{\partial x}{\partial t}=\left(L_{1}^{n}\left[L_{0}[u]\right]\right)_{t}-\left(L_{1}^{n}\left[L_{0}[u]\right]\right)_{x}, \\
\vdots \\
\frac{d^{n} L_{1}^{n}\left[L_{0}[u]\right]}{d t^{n}}=\left(\frac{\partial}{\partial t}-\frac{\partial}{\partial x}\right)^{n} L_{1}^{n}\left[L_{0}[u]\right]
\end{gathered}
$$

в силу условия (2) из (7) и (8) имеем

$$
\Phi_{2}(x)=\varphi_{2 n}(x), \ldots, \Phi_{n}(x)=\varphi_{n+2}(x) .
$$

Тогда уравнение (8) приобретает следующий вид интегро-дифференциального уравнения:

$$
L_{1}^{n}\left[L_{0}[u]\right]=\sum_{i=1}^{n} \varphi_{n+i+1}(x+t) \frac{t^{i-1}}{(i-1) !}+\int_{0}^{t} \frac{(t-s)^{n-1}}{(n-1) !} f(s, x, \vartheta(s)) d s
$$

Аналогично, интегрируя уравнения (10) $n$ раз вдоль второй характеристики, получаем

$$
\begin{aligned}
L_{1}^{n-1}\left[L_{0}[u]\right]=\Phi_{n+2}(x-t)+ & \sum_{j=1}^{n} \int_{0}^{t} \varphi_{n+j+1}(x+s) \frac{s^{j-1}}{(j-1) !} d s+ \\
& +\int_{0}^{t} \frac{(t-s)^{n}}{n !} f(s, x, \vartheta(s)) d s,
\end{aligned}
$$




$$
\begin{gathered}
L_{1}^{n-2}\left[L_{0}[u]\right]=\Phi_{n+3}(x-t)+\Phi_{n+2}(x-t) t+ \\
+\sum_{j=1}^{n} \int_{0}^{t}(t-s) \varphi_{n+j+1}(x+s) \frac{s^{j-1}}{(j-1) !} d s+\int_{0}^{t} \frac{(t-s)^{n+1}}{(n+1 !} f(s, x, \vartheta(s)) d s, \quad(12) \\
\vdots \\
L_{0}[u]=\sum_{i=n+1}^{2 n} \Phi_{i+1}(x-t) \frac{t^{2 n-i}}{(2 n-i) !}+\sum_{j=1}^{n} \int_{0}^{t} \frac{(t-s)^{n-1}}{(n-1) !} \varphi_{n+j+1}(x+s) \frac{s^{j-1}}{(j-1) !} d s+ \\
+\int_{0}^{t} \frac{(t-s)^{2 n-1}}{(2 n-1 !} f(s, x, \vartheta(s)) d s, \quad(13)
\end{gathered}
$$

где $\Phi_{i}(x), i=\overline{n+1,2 n}$ - произвольные непрерывные функции, которые подлежат дальнейшему определению.

Из (11) в силу начального условия (2) имеем $\Phi_{n+1}(x)=\varphi_{n+1}(x)$.

Вдоль третьей характеристики справедливо (9). А вдоль второй характеристики имеем

$$
\begin{gathered}
\frac{d L_{0}[u]}{d t}=\frac{\partial L_{0}[u]}{\partial t}+\frac{\partial L_{0}[u]}{\partial x} \frac{\partial x}{\partial t}=\left(L_{0}[u]\right)_{t}+\left(L_{0}[u]\right)_{x} \\
\vdots \\
\frac{d^{n} L_{0}[u]}{d t^{n}}=\left(\frac{\partial}{\partial t}+\frac{\partial}{\partial x}\right)^{n} L_{0}[u] .
\end{gathered}
$$

Тогда в силу (2) из (12) и (13) получаем

$$
\Phi_{n+2}(x)=\varphi_{n}(x), \ldots, \Phi_{2 n}(x)=\varphi_{2}(x) .
$$

Отсюда имеем следующее квазилинейное интегро-дифференциальное уравнение:

$$
\begin{aligned}
L_{0}[u]=\sum_{i=1}^{n} \varphi_{i+1}(x-t) \frac{t^{i-1}}{(i-1) !}+\sum_{j=1}^{n} \int_{0}^{t} & \frac{(t-s)^{n-1}}{(n-1) !} \varphi_{n+j+1}(x+s) \frac{s^{j-1}}{(j-1) !} d s+ \\
& +\int_{0}^{t} \frac{(t-s)^{2 n-1}}{(2 n-1 !} f(s, x, \vartheta(s)) d s .
\end{aligned}
$$

Интегрируя (15) один раз вдоль линии первой характеристики, с учётом начального условия (2) получаем нелинейное интегральное уравнение Вольтерpa (см. [4,5]):

$$
\begin{aligned}
u(t, x) & \equiv \Theta_{1}(t, x ; u, \vartheta)=\varphi_{1}\left(x-\int_{0}^{t} a\left(s, x, \int_{0}^{T} \int_{-\infty}^{\infty} K(\theta, y) u(\theta, y) d y d \theta\right) d s\right)+ \\
& +\sum_{i=1}^{n} \varphi_{i+1}(x-t) \frac{t^{i}}{i !}+\sum_{j=1}^{n} \int_{0}^{t} \frac{(t-s)^{n-1}}{(n-1) !} \varphi_{n+j+1}(x+s) \frac{s^{j-1}}{(j-1) !} d s+
\end{aligned}
$$




$$
+\int_{0}^{t} \frac{(t-s)^{2 n}}{(2 n) !} f(s, x, \vartheta(s)) d s
$$

где $x$ играет роль параметра.

Функция

$$
\varphi_{1}\left(x-\int_{0}^{t} a\left(s, x, \int_{0}^{T} \int_{-\infty}^{\infty} K(\theta, y) u(\theta, y) d y d \theta\right) d s\right)
$$

является первым интегралом уравнения $u_{t}+a u_{x}=0$ и она постоянна вдоль решения этого уравнения. Производные этой функции вдоль первой характеристики равны нулю и сама функция удовлетворяет данному уравнению.

В (16) также отметим, что функции $\varphi_{2}(x-t), \varphi_{3}(x-t), \ldots, \varphi_{n+1}(x-t)$ являются первыми интегралами уравнения $\left(\frac{\partial}{\partial t}+\frac{\partial}{\partial x}\right)^{n} u(t, x)=0$ и они постоянны вдоль решения этого уравнения. Производные этих функций вдоль второй характеристики равны нулю и сами эти функции удовлетворяют данному уравнению.

А функции $\varphi_{n+2}(x+t), \varphi_{n+3}(x+t), \ldots, \varphi_{2 n+1}(x+t)$ являются первыми интегралами уравнения

$$
\left(\frac{\partial}{\partial t}-\frac{\partial}{\partial x}\right)^{n} u(t, x)=0
$$

и они постоянны вдоль решения этого уравнения. Вдоль третьей характеристики эти функции удовлетворяют данному уравнению.

Исходя из этих соображений покажем, что интегральное уравнение (16) удовлетворяет дифференциальному уравнению в частных производных (1). Путём $(2 n+1)$-кратного дифференцирования из (16) получаем обыкновенное дифференциальное уравнение

$$
\frac{d^{2 n+1} u(t, x)}{d t^{2 n+1}}=f(t, x, \vartheta(t))
$$

где $x$ играет роль параметра.

Так как вдоль третьей характеристики справедливо (9), а вдоль второй характеристики - (14), имеем

$$
\frac{d^{2 n+1} u(t, x)}{d t^{2 n+1}}=\left(\frac{\partial}{\partial t}-\frac{\partial}{\partial x}\right)^{n}\left(\frac{\partial}{\partial t}+\frac{\partial}{\partial x}\right)^{n} L_{0}[u]=\left(\frac{\partial^{2}}{\partial t^{2}}-\frac{\partial^{2}}{\partial x^{2}}\right)^{n}\left(u_{t}+a u_{x}\right)
$$

Отсюда заключаем, что из обыкновенного дифференциального уравнения (17) следует диффференциальное уравнение в частных производных (1).

3. Уравнение для восстанавливаемой функции. Используя условие (4), из (16) получаем

$$
\begin{aligned}
\psi(t) & =\alpha(t) \varphi_{1}\left(x_{0}-\int_{0}^{t} a\left(s, x_{0}, \int_{0}^{T} \int_{-\infty}^{\infty} K(\theta, y) u(\theta, y) d y d \theta\right) d s\right)+ \\
& +\int_{0}^{t} P(t, s)\left(\sum_{i=1}^{n} \varphi_{i+1}\left(x_{0}-t\right) \frac{s^{i}}{i !}+\sum_{j=1}^{n} \varphi_{n+j+1}\left(x_{0}+t\right) \frac{s^{n+j}}{(n+j) !}\right) d s+
\end{aligned}
$$




$$
+\int_{0}^{t} P(t, s) \frac{(t-s)^{2 n+1}}{(2 n+1) !} f\left(s, x_{0}, \vartheta(s)\right) d s, \alpha(t)=\int_{0}^{t} P(t, s) d s
$$

или

$$
\begin{aligned}
& \int_{0}^{t} h(t, s, \vartheta(s)) d s=g(t)- \\
& \quad-\alpha(t) \varphi_{1}\left(x_{0}-\int_{0}^{t} a\left(s, x_{0}, \int_{0}^{T} \int_{-\infty}^{\infty} K(\theta, y) u(\theta, y) d y d \theta\right) d s\right),
\end{aligned}
$$

где

$$
\begin{gathered}
h(t, s, \vartheta(s))=P(t, s) \frac{(t-s)^{2 n+1}}{(2 n+1) !} f\left(s, x_{0}, \vartheta(s)\right) \\
g(t)=\psi(t)-\int_{0}^{t} P(t, s)\left(\sum_{i=1}^{n} \varphi_{i+1}\left(x_{0}-t\right) \frac{s^{i}}{i !}+\sum_{j=1}^{n} \varphi_{n+j+1}\left(x_{0}+t\right) \frac{s^{n+j}}{(n+j) !}\right) d s
\end{gathered}
$$

Относительно восстанавливаемой функции $\vartheta(t)$ уравнение $(18)$ является нелинейным интегральным уравнением Вольтерра первого рода. Его с помощью классических методов невозможно свести к интегральному уравнению Вольтерра второго рода, к которому мы могли бы применять метод последовательных приближений. Уравнение (18) запишем в виде [6]

$$
\begin{aligned}
\vartheta(t)+\int_{0}^{t} G(s) \vartheta(s) d s=\vartheta(t)+g(t)- & \\
-\alpha(t) \varphi_{1}\left(x_{0}-\int_{0}^{t} a\left(s, x_{0}, \int_{0}^{T}\right.\right. & \left.\left.\int_{-\infty}^{\infty} K(\theta, y) u(\theta, y) d y d \theta\right) d s\right)+ \\
& +\int_{0}^{t}[G(s) \vartheta(s)-h(t, s, \vartheta(s))] d s
\end{aligned}
$$

где $0<G(t)$ - произвольная функция такая, что

$$
\exp \left\{-\int_{0}^{t} G(s) d s\right\} \ll 1
$$

Применяя к (19) метод резольвенты ядра $[-G(s)]$, получаем

$$
\begin{gathered}
\vartheta(t)=\vartheta(t)+g(t)-\alpha(t) \varphi_{1}\left(x_{0}-\int_{0}^{t} a\left(s, x_{0}, \int_{0}^{T} \int_{-\infty}^{\infty} K(\theta, y) u(\theta, y) d y d \theta\right) d s\right)+ \\
+\int_{0}^{t}[G(s) \vartheta(s)-h(t, s, \vartheta(s))] d s-\int_{0}^{t} G(s) \exp \{-\mu(t, s)\}\{\vartheta(s)+g(s)- \\
\quad-\alpha(s) \varphi_{1}\left(x_{0}-\int_{0}^{s} a\left(\theta, x_{0}, \int_{0}^{T} \int_{-\infty}^{\infty} K(\xi, y) u(\xi, y) d y d \xi\right) d \theta\right)+ \\
\left.+\int_{0}^{s}[G(\theta) \vartheta(\theta)-h(s, \theta, \vartheta(\theta))] d \theta\right\} d s, \quad(20)
\end{gathered}
$$


где

$$
\mu(t, s)=\int_{s}^{t} G(\theta) d \theta, \quad \mu(t, 0)=\mu(t) .
$$

После несложных преобразований из (20) имеем

$$
\begin{aligned}
\vartheta(t) \equiv \Theta_{2}(t ; u, \vartheta)=\{\vartheta(t)+g(t)- \\
-\alpha(t) \varphi_{1}\left(x_{0}-\int_{0}^{t} a\left(s, x_{0}, \int_{0}^{T} \int_{-\infty}^{\infty} K(\theta, y) u(\theta, y) d y d \theta\right) d s\right)+ \\
\left.+\int_{0}^{t}[G(s) \vartheta(s)-h(t, s, \vartheta(s))] d s\right\} \exp \{-\mu(t)\}+ \\
+\int_{0}^{t} G(s) \exp \{-\mu(t, s)\}\{g(t)-g(s)+\vartheta(t)-\vartheta(s)- \\
\quad-\alpha(t) \varphi_{1}\left(x_{0}-\int_{0}^{t} a\left(s, x_{0}, \int_{0}^{T} \int_{-\infty}^{\infty} K(\theta, y) u(\theta, y) d y d \theta\right) d s\right)+ \\
+\alpha(s) \varphi_{1}\left(x_{0}-\int_{0}^{s} a\left(\theta, x_{0}, \int_{0}^{T} \int_{-\infty}^{\infty} K(\xi, y) u(\xi, y) d y d \xi\right) d \theta\right)+ \\
\left.+\int_{0}^{t}[G(s) \vartheta(s)-h(t, s, \vartheta(s))] d s-\int_{0}^{s}[G(\theta) \vartheta(\theta)-h(s, \theta, \vartheta(\theta))] d \theta\right\} d s .
\end{aligned}
$$

Уравнение (18) при начальном условии (4) эквивалентно уравнению (21).

4. Разрешимость обратной задачи (1)-(4). Итак, мы получаем, что разрешимость обратной задачи (1)-(4) эквивалентна разрешимости следующей системы нелинейных интегральных уравнений:

$$
\left\{\begin{array}{l}
u(t, x) \equiv \Theta_{1}(t, x ; u, \vartheta) \\
\vartheta(t) \equiv \Theta_{2}(t ; u, \vartheta)
\end{array}\right.
$$

где $x$ играет роль параметра.

Для произвольной непрерывной в области $D$ функции $h(t, x)$ норму вводим следующим образом:

$$
\|h(t, x)\|=\max _{(t, x) \in D}|h(t, x)|
$$

где $x$ играет роль параметра. Аналогично вводится норма для функции одной переменной.

Теорема. Пусть выполняются следующие условия:

1) $\left|\varphi_{i}(x)\right| \leqslant M_{i}, 0<\sum_{i=1}^{2 n+1} M_{i} \frac{T^{i}}{i !} \leqslant \Delta_{0}<\infty$;

2) $f(t, x, \vartheta) \in \operatorname{Bnd}\left(M_{0}(t)\right) \cap \operatorname{Lip}\left\{\left.L_{0}(t)\right|_{\vartheta}\right\}$;

3) $0<\int_{0}^{T} \frac{(T-s)^{2 n}}{(2 n) !} M_{0}(s) d s \leqslant \Delta_{1}<\infty$; 
4) $0<\int_{0}^{T} \frac{(T-s)^{2 n}}{(2 n) !} L_{0}(s) d s \leqslant \Delta_{2}<\infty$;

5) $\varphi_{1}(x) \in \operatorname{Lip}\left\{\left.L_{1}\right|_{x}\right\}, 0<L_{1}<\infty$;

6) $a(t, x, z) \in \operatorname{Lip}\left\{\left.L_{2}(t)\right|_{z}\right\}$,

$$
0<L_{1} \int_{0}^{T} L_{2}(t) \int_{0}^{T} \int_{-\infty}^{\infty} K(s, y) d y d s d t \leqslant \Delta_{3}<\infty
$$

7) $\rho<1$, где $\rho=\max \left\{\beta_{1} ; \beta_{2}\right\}, \beta_{2}=\max \left\{\Delta_{3} ;\|\alpha(t)\| \Delta_{3} M_{0}\right\}$,

$$
\begin{aligned}
& \beta_{1}=\max \left\{\Delta_{2} ;\left(1+\int_{0}^{T}\|G(t)\| d t+\|P(t, s)\| \frac{T \Delta_{2}}{2 n+1}\right) M_{0}\right\}, \\
& M_{0}=\max _{t \in D_{T}}\left\{\exp \{-\mu(t)\}+2 \int_{0}^{t} G(s) \exp \{-\mu(t, s)\} d s\right\} \ll 1 .
\end{aligned}
$$

Тогда обратная задача (1)-(4) имеет единственное решение в области D.

До ка з а те ль с т в о. Воспользуемся методом последовательных приближений. Рассмотрим следующий итерационный процесс:

$$
\begin{cases}u_{0}(t, x)=0, & u_{k+1}(t, x) \equiv \Theta_{1}\left(t, x ; u_{k}, \vartheta_{k}\right), \\ \vartheta_{0}(t)=0, & \vartheta_{k+1}(t) \equiv \Theta_{2}\left(t ; u_{k}, \vartheta_{k}\right), \quad k=0,1,2, \ldots,\end{cases}
$$

где $x$ играет роль параметра. Тогда в силу условий теоремы из (23) получаем, что справедливы следующие оценки:

$$
\begin{aligned}
& \left\|u_{1}(t, x)-u_{0}(t, x)\right\| \leqslant \sum_{i=1}^{2 n+1} M_{i} \frac{t^{i}}{i !}+\int_{0}^{t} \frac{(t-s)^{2 n}}{(2 n) !} M_{0}(s) d s \leqslant \Delta_{0}+\Delta_{1} ; \\
& \left\|u_{k+1}(t, x)-u_{k}(t, x)\right\| \leqslant \\
& \leqslant L_{1} \int_{0}^{t} L_{2}(s) \int_{0}^{T} \int_{-\infty}^{\infty} K(\theta, y)\left\|u_{k}(\theta, y)-u_{k-1}(\theta, y)\right\| d y d \theta d s+ \\
& +\int_{0}^{t} \frac{(t-s)^{2 n}}{(2 n) !} L_{0}(s)\left\|\vartheta_{k}(s)-\vartheta_{k-1}(s)\right\| d s \leqslant \\
& \leqslant \Delta_{3}\left\|u_{k}(t, x)-u_{k-1}(t, x)\right\|+\Delta_{2}\left\|\vartheta_{k}(t)-\vartheta_{k-1}(t)\right\| ; \\
& \left\|\vartheta_{1}(t)-\vartheta_{0}(t)\right\| \leqslant\left(\|g(t)\|+\|\alpha(t)\| M_{1}+\int_{0}^{t}\|h(t, s, 0,0)\| d s\right)\{\exp \{-\mu(t)\}+ \\
& \left.+2 \int_{0}^{t} G(s) \exp \{-\mu(t, s)\}\left(\|g(t)\|+\|\alpha(t)\| M_{1}+\int_{0}^{t}\|h(t, s, 0,0)\| d s\right) d s\right\} \leqslant \\
& \leqslant\left(\|g(t)\|+\|\alpha(t)\| M_{1}+\int_{0}^{T}\|h(T, s, 0,0)\| d s\right) M_{0}, \\
& M_{0}=\max _{t \in D_{T}}\left\{\exp \{-\mu(t)\}+2 \int_{0}^{t} G(s) \exp \{-\mu(t, s)\} d s\right\} \ll 1 ;
\end{aligned}
$$




$$
\begin{gathered}
\left\|\vartheta_{k+1}(t)-\vartheta_{k}(t)\right\| \leqslant \\
\leqslant\|\alpha(t)\| M_{0} L_{1} \int_{0}^{t} L_{2}(s) \int_{0}^{T} \int_{-\infty}^{\infty} K(\theta, y)\left\|u_{k}(\theta, y)-u_{k-1}(\theta, y)\right\| d y d \theta d s+ \\
+\left\{1+\int_{0}^{t}\|G(s)\| d s+\int_{0}^{T}\|P(t, s)\| \frac{(T-s)^{2 n+1}}{(2 n+1) !} L_{0}(s) d s\right\} M_{0}\left\|\vartheta_{k}(t)-\vartheta_{k-1}(t)\right\| \leqslant \\
\leqslant\|\alpha(t)\| \Delta_{3}\left\|u_{k}(t, x)-u_{k-1}(t, x)\right\|+ \\
+\left\{1+\int_{0}^{T}\|G(t)\| d t+\|P(t, s)\| \frac{T \Delta_{2}}{2 n+1}\right\} M_{0}\left\|\vartheta_{k}(t)-\vartheta_{k-1}(t)\right\| .
\end{gathered}
$$

Примем следующие обозначения:

$$
\begin{gathered}
\beta_{1}=\max \left\{\Delta_{2} ;\left(1+\int_{0}^{T}\|G(t)\| d t+\|P(t, s)\| \frac{T \Delta_{2}}{2 n+1}\right) M_{0}\right\}, \\
\beta_{2}=\max \left\{\Delta_{3} ;\|\alpha(t)\| \Delta_{3} M_{0}\right\}, \quad \rho=\max \left\{\beta_{1} ; \beta_{2}\right\} .
\end{gathered}
$$

Тогда из (25) и $(27)$ имеем

$$
\left\|U_{k+1}(t, x)-U_{k}(t, x)\right\| \leqslant \rho\left\|U_{k}(t, x)-U_{k-1}(t, x)\right\|,
$$

где $\left\|U_{k}(t, x)-U_{k-1}(t, x)\right\| \equiv\left\|u_{k}(t, x)-u_{k-1}(t, x)\right\|+\left\|\vartheta_{k}(t)-\vartheta_{k-1}(t)\right\|$.

Из оценок $(24),(26)$ и (28) следует, что операторы в правой части системы (22) являются сжимающими. Следовательно, обратная задача (1)-(4) имеет единственное решение в области $D$.

\section{БИБЛИОГРАФИЧЕСКИЙ СПИСОК}

1. A. М. Денисов, Введение в теорию обратных задач. М.: МГУ, 1994. 285 с. [A. M. Denisov, Introduction to the theory of inverse problem. Moscow: Lomonosov Moscow State University, 1994. 285 pp.]

2. В. Г. Романов, Обратные задачи для математической физики. М.: Наука, 1984. 264 с. [V. G. Romanov, Inverse problem for mathematical physics. Moscow: Nauka, 1984. 264 pp.]

3. М. М. Лаврентъев, Л. Я. Савельев, Линейные операторы и некорректные задачи. М.: Наука, 1999. 330 с. [M. M. Lavrent'ev, L. Ya. Savel'ev, Linear operators and ill-posed problems. Moscow: Nauka, 1999. 330 pp.]

4. Т. К. Юлдашев, "Об обратной задаче для квазилинейного уравнения в частных производных первого порядка" // Вестн. Томск. гос. ун-та. Матем. и мех., 2012. № 2 . C. 56-62. [T. K. Yuldashev, "On the inverse problem for the quasilinear partial differential equation of the first order" // Vestn. Tomsk. Gos. Univ. Mat. Mekh., 2012. no. 2. Pp. 56-62].

5. Т. К. Юлдашев, "Об обратной задаче для системы квазилинейных уравнений в частных производных первого порядка" // Вестн. ЮУрГУ. Сер. Математика. Механика. Физика, 2012. Т. 6, №11(270). С. 35-41. [T. K. Yuldashev, "On the inverse problem for a system of quasi-linear partial differential equations of the first order" // Vestn. YuUrGU. Ser. Matematika. Mekhanika. Fizika, 2012. Vol.6, no.11(270). Pp. 35-41].

6. Т. К. Юлдашев, "Неявное эволюционное интегральное уравнение Вольтерра первого рода с нелинейным интегральным отклонением" // Вестн. Сам. гос. техн. ун-та. Сер. Физ.-мат. науки, 2009. № 2(19). C. 38-44. [T. K. Yuldashev, "Nonexplicit evolution Volterra integral equation of the first kind with nonlinear integral delay" // Vestn. Samar. Gos. Tekhn. Univ. Ser. Fiz.-Mat. Nauki, 2009. no. 2(19). Pp. 38-44].

Поступила в редакцию 06/XI/2012;

в окончательном варианте - 14/III/2013. 
MSC: 35K70, 35R30

\section{INVERSE PROBLEM FOR QUAZILINEAR PARTIAL INTEGRO-DIFFERENTIAL EQUATIONS OF HIGHER ORDER}

\section{T. K. Yuldashev, A.I. Seredkina}

M. F. Reshetnev Siberian State Aerospace University,

31, pr. "Krasnoyarski Rabochiy", Krasnoyarsk, 660014, Russia.

E-mails: tursunbay@rambler.ru, anytik888@yandex.ru

A method of studying an inverse problem for the some classes of quasilinear partial integro-differential equation of the higher order is proposed. A theorem on the existence and uniqueness of the solution of this problem is proved.

Key words: inverse problem, quasilinear equation, superposition of differential operator, characteristics method, existence and uniqueness of the solution.

Original article submitted 06/XI/2012; revision submitted 14/III/2013.

Tursun K. Yuldashev (Ph. D. Phys. \& Math.), Doctoral Candidate, Associate Professor, Dept. of Higher Mathematics. Ann I. Seredkina, Graduate Student, Ingineer, Dept. of Higher Mathematics. 